\title{
A Diagnostic Program of Vascular Tumor and Vascular Malformations in Children According to Modern Classification
}

\author{
Iryna Benzar*
}

\section{ABSTRACT}

The aim of the study was to analyze the cohort of inpatient children with vascular anomalies according to the globally accepted classification introduced by the ISSVA. Methods: The study included 205 inpatient children within the time period of the years $2010-2015$. Types of vascular anomalies (VAs), age of patients, diagnostic procedures, and anatomical localization of VAs were analyzed. Results: 65 patients of first year of life had vascular tumors, with prevalence of infantile hemangiomas (IHs) in $57(87.7 \%)$ patients. 45 children had IHs localized within soft tissues, whereas 7 patients suffered from IHs of the liver, and 5 children from IHs of the respiratory tract. Most patients with soft tissue IHs were diagnosed only with ultrasound; CT or MRI diagnostics were performed on 5 (8.8\%) patients, and biopsy was carried out in 2 (4.4\%) children. Vascular malformations (VM) were diagnosed in 140 (68.3\%) patients. Ultrasound investigation (US) was the screening method. MRI was performed to confirm the diagnosis of low-flow VM, whereas for high-flow VM CT angiography and selective angiography were useful. Venous malformations were diagnosed in 17 (12.1\%) patients, and 112 (80.0\%) had cystic LM, among them children under the age of 2 years prevailed. Arteriovenous malformations were diagnosed in 5 (3.8\%) patients, ages 2-14 years. Conclusions: Clinical manifestations of vascular anomalies have clear age features. Among hospitalized children vascular tumors add up to $31.7 \%$ and VM - up to $68.3 \%$.

\section{KEYWORDS}

vascular anomalies; vascular tumors; vascular malformations; ISSVA Classification for vascular anomalies

\section{AUTHOR AFFILIATIONS}

Pediatric Surgery Department, Bogomolets National Medical University, Kyiv, Ukraine

*Corresponding author: 10G Vitryani Gory str, apt. 32, Kyiv, 04123, Ukraine; e-mail: ira_benzar@yahoo.com, iryna.benzar@nmu.ua

Received: 3 November 2016

Accepted: 12 January 2017

Published online: 26 May 2017

Acta Medica (Hradec Králové) 2017; 60(1): 19-26

https://doi.org/10.14712/18059694.2017.47

(c) 2017 The Author. This is an open-access article distributed under the terms of the Creative Commons Attribution License (http://creativecommons.org/licenses/by/4.0), which permits unrestricted use, distribution, and reproduction in any medium, provided the original author and source are credited. 


\section{BACKGROUND}

The field of vascular anomalies (VAs) is considered to be a special focus of pediatric surgeons, and it has shown a rapid development during the last decade. Patients with VAs are always difficult to identify and clearly describe, given the large number of pathology and diverse terminology that is used in many medical institutions. Despite long-standing efforts to promulgate standard classification, nomenclature terminology of vascular anomalies is still confusing (1).

The long history of such confusing terminology complicated the communication between physicians of different specialties. The symbol of nomenclature inconsistencies is the term "hemangioma" which even now is widely used to describe various vascular lesions (2).

Because many VAs, including most uncomplicated $\mathrm{IH}$, capillary local spots, etc., do not pose a threat to the life or health of the patient and do not require hospital treatment, these children may be under the supervision of outpatient pediatricians, dermatologists or surgeons. Other diseases are life threatening, have progressing outcome and potential dangers of complications. Introduction of the uniform classification will be the first step towards correct diagnosis and appropriate treatment.

The aim of this study was to analyze the inpatient group of patients with VAs and classify them according to the globally accepted classification introduced by the ISSVA (International Society for the Study of Vascular Anomalies) and to describe the diagnostic methods and findings used to distinguish separate groups.

The diagnosis of VAs was made according to the updated ISSVA (International Society for the Study of Vascular Anomalies) Classification of Vascular Anomalies that was adopted at the workshop in Melbourne, Australia (April 2014) (3). One of the goals of the ISSVA is to achieve a uniform classification (4). The new ISSVA classification scheme is based on the fundamental separation of vascular anomalies into those lesions with a proliferative component (named "vascular tumors") versus relatively static "vascular malformations," following Mulliken and Glowacki’s (5).

\section{MATERIAL AND METHODS}

The retrospective study included 205 inpatient children with VAs in the time period from year 2010 to 2015, ages 1 month to 17 years. The age of patients, types of anomalies and their anatomical locations, type and amount of diagnostic procedures for the referred patient were analyzed. The first line investigation of all patients was ultrasound investigation with a 12-3 MHz linear transducer in gray scale mode, color Doppler scan (CDS) and partially in Doppler mode. The additional investigations included computed tomography (CT), both native and intravenous enhanced; magnetic resonance imaging (MRI), biopsy and selective angiography.

\section{RESULTS}

Vascular tumors were diagnosed in 65 patients with IHs predominance $(n=57 ; 87.7 \%)$. Soft tissue IHs were diagnosed in 45 children, 7 children had IHs of the liver and 7 children had IHs of the respiratory tract. The age of children with IHs at the time of hospitalization ranged from 1 to 11 months, with the age average of 3.8 months. The indications for hospitalization were IHs of difficult anatomical localization, which required further examination to clarify the underlying diagnosis, and complicated IHs, which needed hospital treatment.

To confirm the diagnosis of IHs of the skin, subcutaneous tissue, muscles, visible mucous membranes, ultrasound mode gray scale and color Doppler scans were used. The US features of IHs were determined by its growth phase. In proliferative phase they were presented as a hypoechoic lobular mass, CDS mode revealed maximum microcirculation and non-vascularized tissue was absent (Fig. 1).

In maturation and regression phases the structure of the IHs becomes hyperechoic, in CDS mode increasing ectatic drainage veins and declining arterialization of drainage veins were visualized (Fig. 2).

If the typical skin lesions were absent, and in cases of localization of IHs in deep tissues, additional method of visualization were used in $5(8.8 \%)$ patients. It was intravenous enhanced CT (Fig. 3) in two cases, MRI in three children, and biopsy (Fig. 4) performed in two clinically unclear cases.

To confirm the diagnosis for IHs of the liver intravenous contrast-enhance CT $(n=6)$ and MRI $(n=1)$ were performed. Three children had multiple skin hemangiomas and multiple hepatic hemangiomas. Diagnosis for IHs of the respiratory system in children with respiratory failure was determined by the results of laryngobronchoscopy. To determine the borders of lesions and the spreading throughout the mediastinum, an intravenous contrast-enhanced CT $(n=4)$ and MRI $(n=1)$ were performed.

Thrombocytopenia, the so-called Kasabach-Merritt phenomenon occurred only in two children with vascular tumors: in a newborn girl with Kaposiform hemangioendothelioma (platelet count $8-10 \times 10^{3} / \mathrm{mL}$ ) and moderate thrombocytopenia in a 4-month-old girl with tufted angioma (platelet count $80-90 \times 10^{3} / \mathrm{mL}$ ). There were no coagulation disorders in patients with IHs.

Rapidly involuting congenital hemangiomas (RICH) were diagnosed in 5 patients. Localizations of RICHs were at the head and neck region $(\mathrm{n}=2)$, upon extremities $(n=2)$ (Fig. 5), and in the liver $(n=1)$.

Vascular malformations (VM) were diagnosed in 140 (68.3\%) of the hospitalized patients. During the primary investigation of patients with vascular malformations US visualization with Doppler mode allowed to differentiate fast flow and slow flow malformations. To confirm the diagnosis of slow flow vascular malformation MRI was performed. High flow vascular malformations were visualized by CT angiography and selective angiography.

VM were diagnosed in 17 children ages from 1 month to 17 years, the average age being 7.4 years. The percentage rate of VM was $12.1 \%$ out of all vascular malformations. 

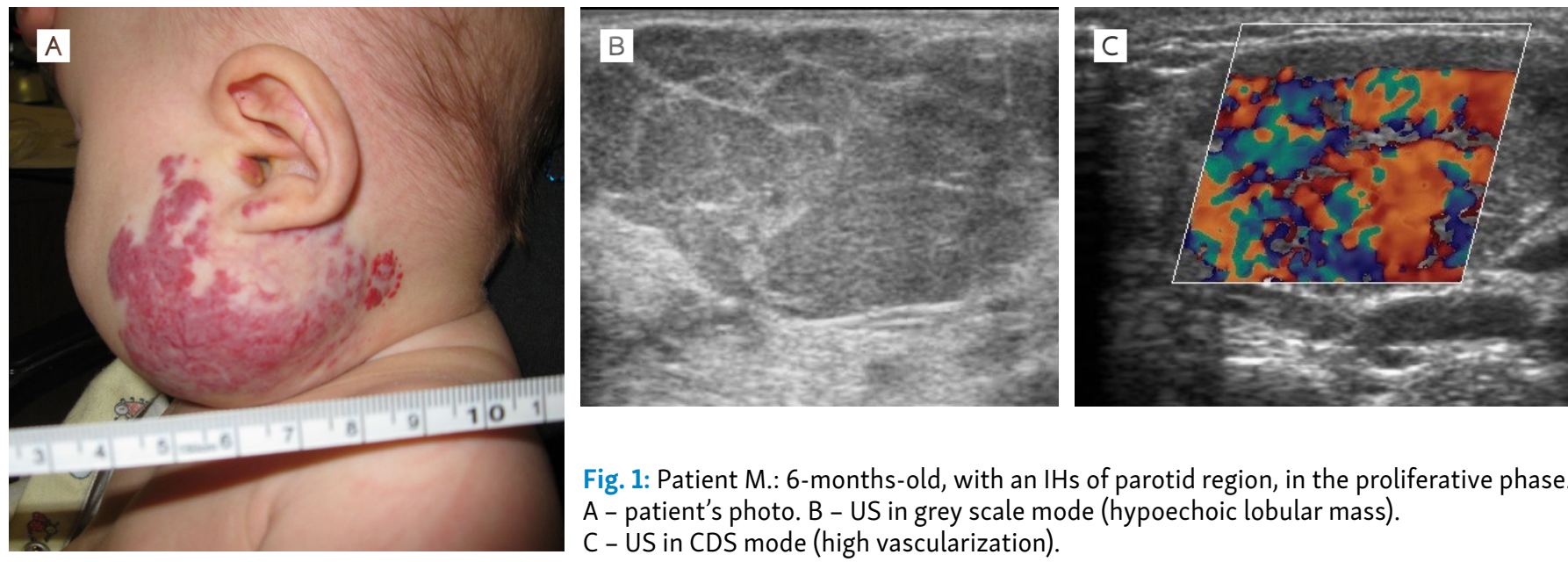

Fig. 1: Patient M.: 6-months-old, with an IHs of parotid region, in the proliferative phase. A - patient's photo. B - US in grey scale mode (hypoechoic lobular mass). $C$ - US in CDS mode (high vascularization).
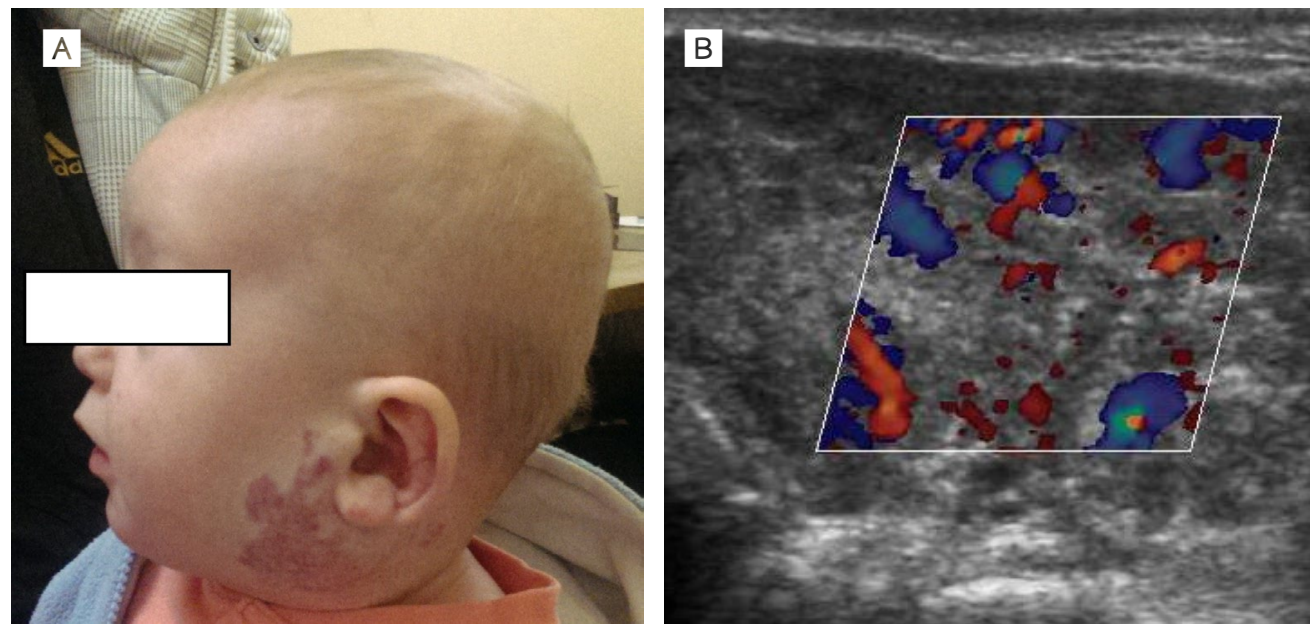

Fig. 2: Patient M.: 11-months-old, with an IHs of the parotid region, in the maturation phase. A - patient's photo. B - US in CDS mode (circumscribed hypersonoric area, ectatic veins on the background of non-vascularized tissue).

At US, VM were represented as a compressible fluid-filled cavitary septate lesions with inhomogeneous echogenicity, its lumen could often reveal pathognomonic phleboliths with acoustic shadow. On MRI VM were visualized as single or multiple masses, lobular or cavernous structures sometimes with infiltration of the surrounding tissues. Loss of signal was the sign of clots or phleboliths (Fig. 6)

D-dimer level elevation more than three times occurred in $14(82.4 \%)$ patients with VMs. Three of them had low fibrinogen level.

Most children ( $\mathrm{n}=112$ ) had cystic lymphatic malformations (LM). The percentage rate of LM among the patients with vascular malformations was $80 \%$. In $12(10.7 \%)$ patients the diagnosis was made at an US screening investigation. Immediately after birth, the clinical manifestations were found in 49 (43.75\%) children. Before the age of two years LM were diagnosed in 35 (31.25\%) patients. Overall, in $85.7 \%$ of patients with cystic LM, primary symptoms were revealed during first two years of life, and in the other 16 children (14.3\%) these symptoms were found at different age periods, particularly in 9 children $(8.0 \%)$ ages 2 to 5 years, in 4 children (3.6\%) from 5 to 12 years, and in $3(2.7 \%)$ of those over 12 years of age.

We usually performed MRI before treatment. According to the results of MRI, the size and structure of LM, and also their topographical relationship with neighboring organs and tissues were determined. LM were classified as macrocystic, microcystic, and mixed (Fig. 7).

Arteriovenous malformations (AVM) were diagnosed in $5(2.4 \%)$ children. The patients' age ranged from 2 to 14

Tab. 1: The anatomical localization of vascular tumors $(n=65)$.

\begin{tabular}{|l|l|l|l|l|l|}
\hline & Head/neck & Trunk & Extremity & Visceral & Total / percentage \\
\hline Total & $26(40.0 \%)$ & $4(6.2 \%)$ & $22(33.8 \%)$ & $13(20.0 \%)$ & $65(100 \%)$ \\
\hline IHs & $24(42.1 \%)$ & $4(7.0 \%)$ & $17(29.8 \%)$ & $12(21.1 \%)$ & $57(87.7 \%)$ \\
\hline Congenital hemangiomas & $2(40.0 \%)$ & & $2(40.0 \%)$ & $1(20.0 \%)$ & $5(7.7 \%)$ \\
\hline $\begin{array}{l}\text { Tufted angioma / } \\
\text { Kaposiform } \\
\text { hemangioendothelioma }\end{array}$ & & $3(100 \%)$ & & 3 & \\
\hline
\end{tabular}



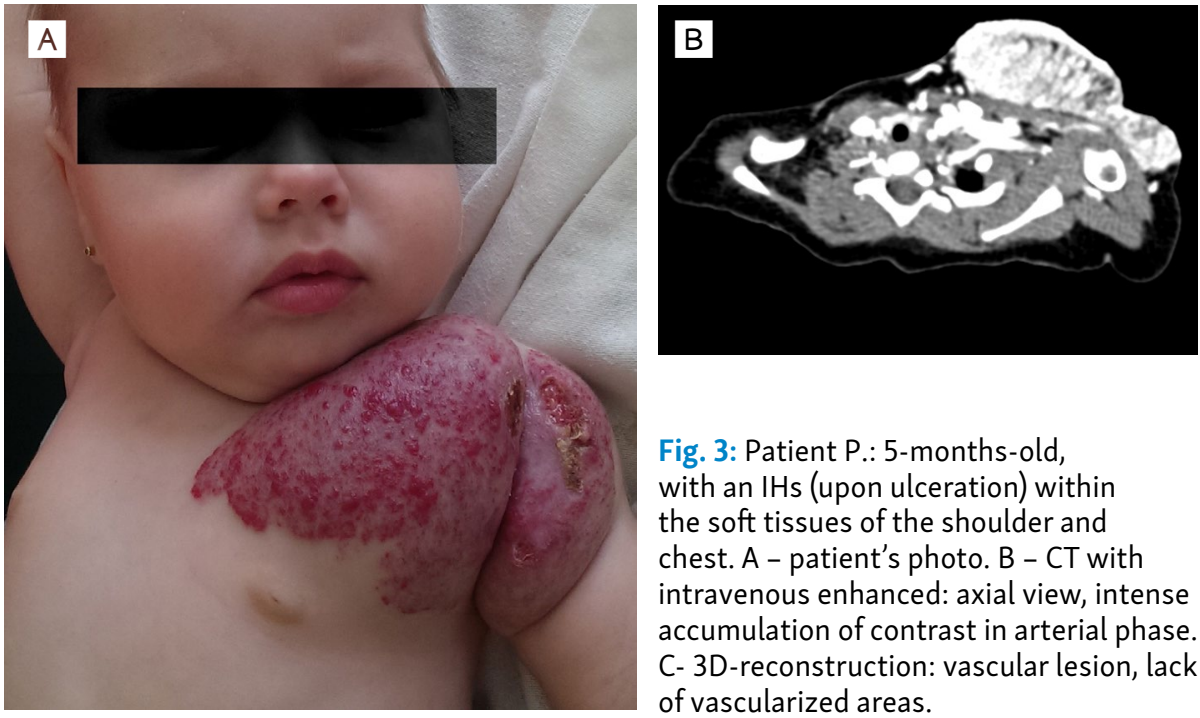

Fig. 3: Patient P.: 5-months-old, with an IHs (upon ulceration) within the soft tissues of the shoulder and chest. A - patient's photo. B - CT with intravenous enhanced: axial view, intense

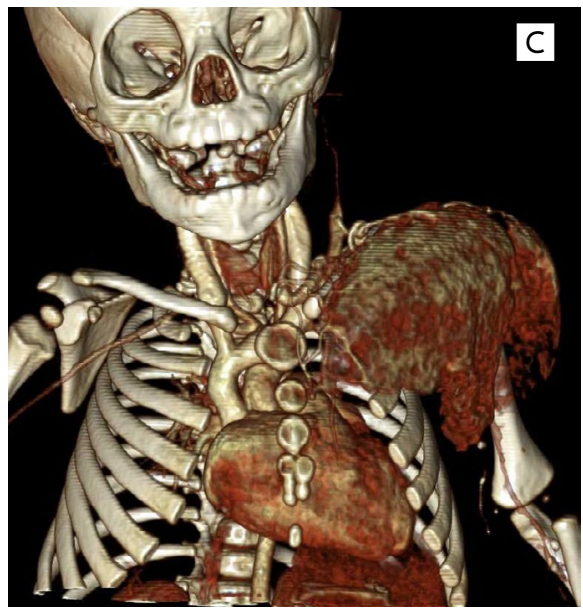
accumulation of contrast in arterial phase. C- 3D-reconstruction: vascular lesion, lack of vascularized areas.
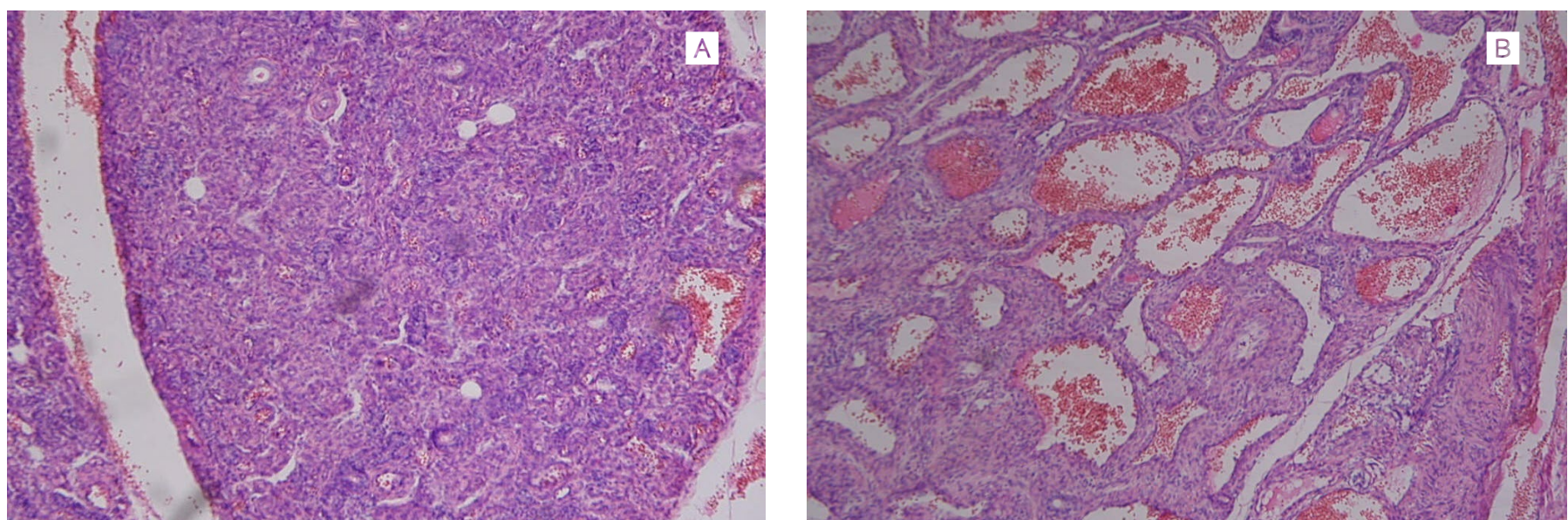

Fig. 4: Light microscopy, hematoxylin-eosin staining, $\times 10$. A - IH, proliferative phase: dense clusters of cells, lumens difficult to discern. $\mathrm{B}-\mathrm{IH}$, maturation phase: dilated vascular channels, small foci of proliferation.

years, with the age average of 8.2 years. AVM were often difficult to distinguish from other vascular malformations and some vascular tumors. AVM at US in CDS mode had the same features as IHs in proliferative phase, however vascularization intensity did not decrease with age (Fig. 8).

CT angiography was performed in all patients. This study allowed the visualization of both normal and pathologically altered vessels and their connections. Selective angiography was performed in three patients immediately before treatment. According to the results of investigation, features of the anatomical location of vascular tumors and vascular malformations were established, and they are presented in Tab. 1 and Tab. 2.

\section{DISCUSSION}

A worrying sign is that more than half of patients with VAs come to specialized centers with an incorrect diagnosis (6). The reason for this may be lack of consistency in

Tab. 2: The anatomical localization of vascular malformations $\left(n=134^{*}\right)$.

\begin{tabular}{|l|l|l|l|l|l|}
\hline & Head/neck & Trunk & Extermity & Viscera & Total / percentage \\
\hline Total & $74(55.2 \%)$ & $19(14.2 \%)$ & $16(11.9 \%)$ & $25(18.7 \%)$ & $134(100 \%)$ \\
\hline LM & $67(59.8 \%)$ & $13(11.6 \%)$ & $9(8.1 \%)$ & $23(20.5 \%)$ & $112(83.5 \%)$ \\
\hline VM & $3(17.6 \%)$ & $6(35.3 \%)$ & $6(35.3 \%)$ & $2(11.8 \%)$ & $17(12.7 \%)$ \\
\hline AVM & $4(80.0 \%)$ & & $1(20.0 \%)$ & & $5(3.8 \%)$ \\
\hline
\end{tabular}

* Combined vascular malformations are not taken into account because they affect different anatomical regions. 

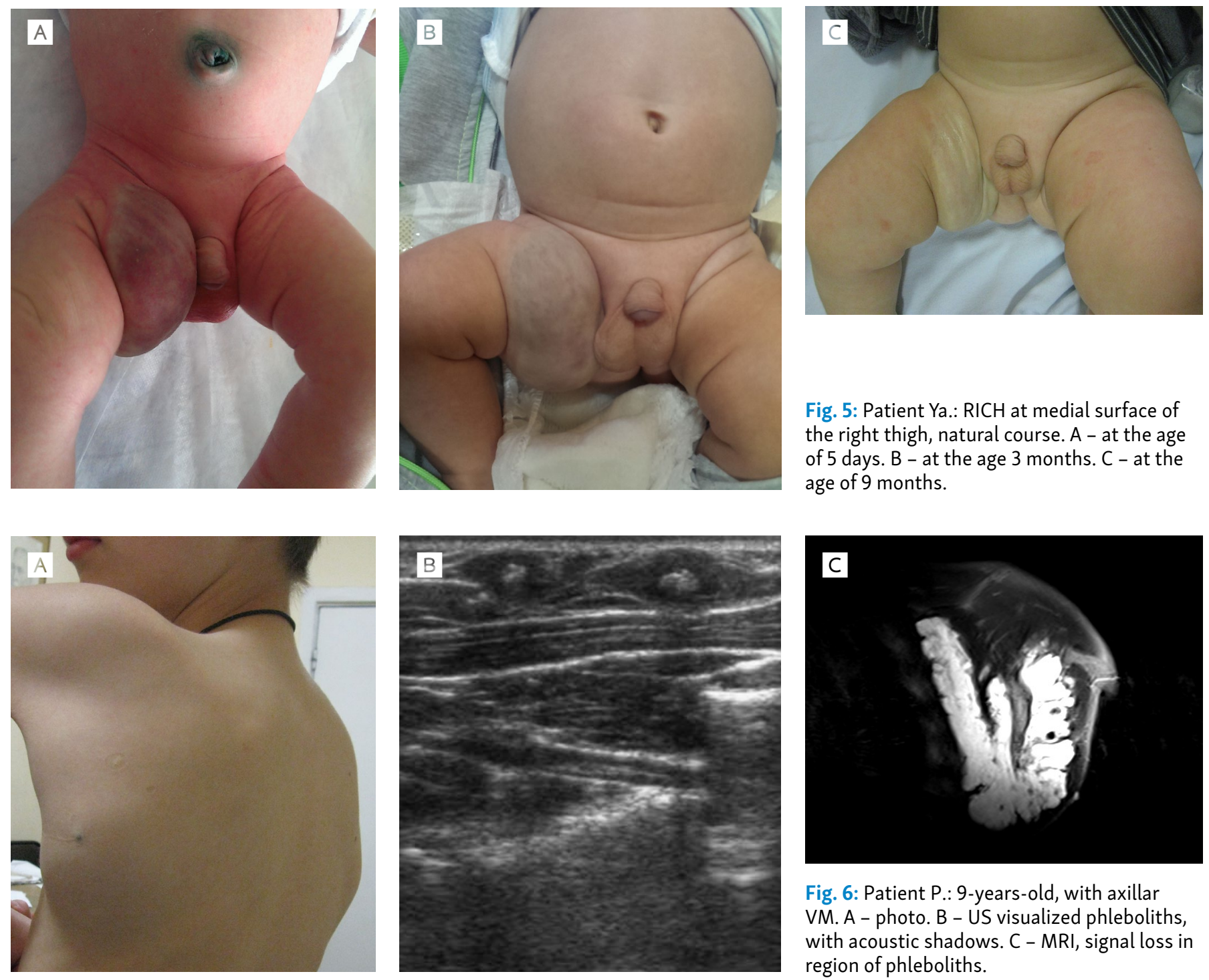

Fig. 5: Patient Ya.: RICH at medial surface of the right thigh, natural course. $A$ - at the age of 5 days. $B$ - at the age 3 months. $C$ - at the age of 9 months.

Fig. 6: Patient P.: 9-years-old, with axillar VM. A - photo. B - US visualized phleboliths, with acoustic shadows. C - MRI, signal loss in region of phleboliths.

terminology and use of inappropriate imaging techniques for diagnosis. The study confirms that VM are much more difficult to diagnose than vascular tumors, and require the use of a wide range of additional research methods. Although VM ( 1 in 200 people) occur 10 times less commonly than vascular tumors ( 1 in 20 people) (7), they account for over two thirds of patients who require hospitalization.

In most cases the diagnosis of IHs of the skin is made only according to the results of physical examination. However in some cases (for example, normal skin and a mass in deep soft tissues, and atypical cutaneous manifestations, complicated localization) diagnosis based solely on the examination of the patient may lead to diagnostic and, subsequently, to tactical mistakes (8). Early diagnosis is very important to determine the therapeutic tactics whether active or expectant (9). MRI in patients with IHs of the soft tissues is used as a reserved method of visualization only in unusual cases, atypical clinical presentation, and in patients with large IHs existing at birth, to determine the borders of the lesion and the characteristics of tissue and vascularization of effected area.

Congenital hemangiomas complete their growth and maturation prenatally and should be clearly differenti- ated from IHs. Congenital hemangiomas, both with rapid involution and without involution, are solitary tumor, frequently located on the extremities near large joints or on the scalp (10). An US examination shows arteries and veins that pass vertically towards the surface of the tumor (11). Congenital hemangiomas are rare tumors, with various, sometimes not classic symptoms, that can mask other diseases, thus observation in medical centers is required.

Kaposiform hemangioendothelioma and tufted angioma are rare vascular tumors that occur in early childhood and can occasionally occur in adults. Both tumors are characterized by local aggressive growth and may be accompanied by a Kasabach-Merritt phenomenon, which is a coagulopathy, associated with severe thrombocytopenia and hypofibrinogenemia $(12,13)$.

Historically in young children VM are mistaken for IHs, and labeled with an outdated term "cavernous hemangiomas". While the most common IHs are specific tumors of the childhood age with spontaneous involution, VM are remnants of embryonic tissue that never disappears spontaneously (14). A clear understanding of these critical factors and the exact wording of the diagnosis are essential for successful treatment (15). According to published 

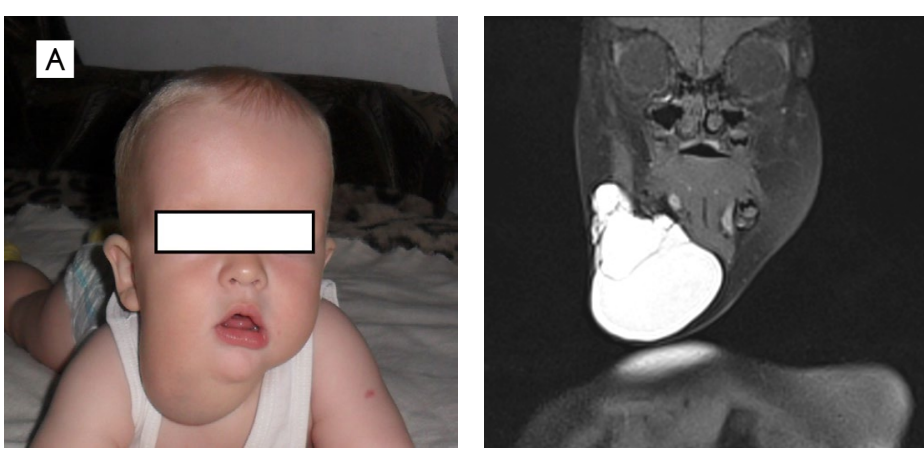

Fig. 7: Cystic LM (patient's photo and MRI). A- macrocystic LM. $B$ - mixed LM. C-microcystic LM.

data, VM are the most common congenital vascular malformations with a frequency of 1-2:10,000 (16). However, in pediatric clinic, the percentage of VM was only $12.1 \%$ out of patients with vascular malformations. Abnormal veins sometimes are visible already at an early age, but the peak of clinical manifestations accounts for about 20 years, at least $10 \%$ of limbs VM are visually seen before the adolescent age (17). This may explain the discrepancy of statistical data in the general population and pediatric clinic. As well there are different statistics in different age groups. In particular, large lymphatic malformations with the compression of adjacent organs dominates among newborns, IHs dominate among children of the first three years of life, and LM causing primarily cosmetic deformity significantly prevail among children older than three years of age.

MRI sequences routinely are used to image vascular malformations. T1 and T2 weighted MRI are the "gold standard" for investigating slow flow vascular malformations, which is necessary to performed before starting treatment. VM are visualized as a single or multiple lobular or cavernous masses, sometimes with infiltrative growth, isointensity or hypointensity on $\mathrm{T} 1$ weighted, and
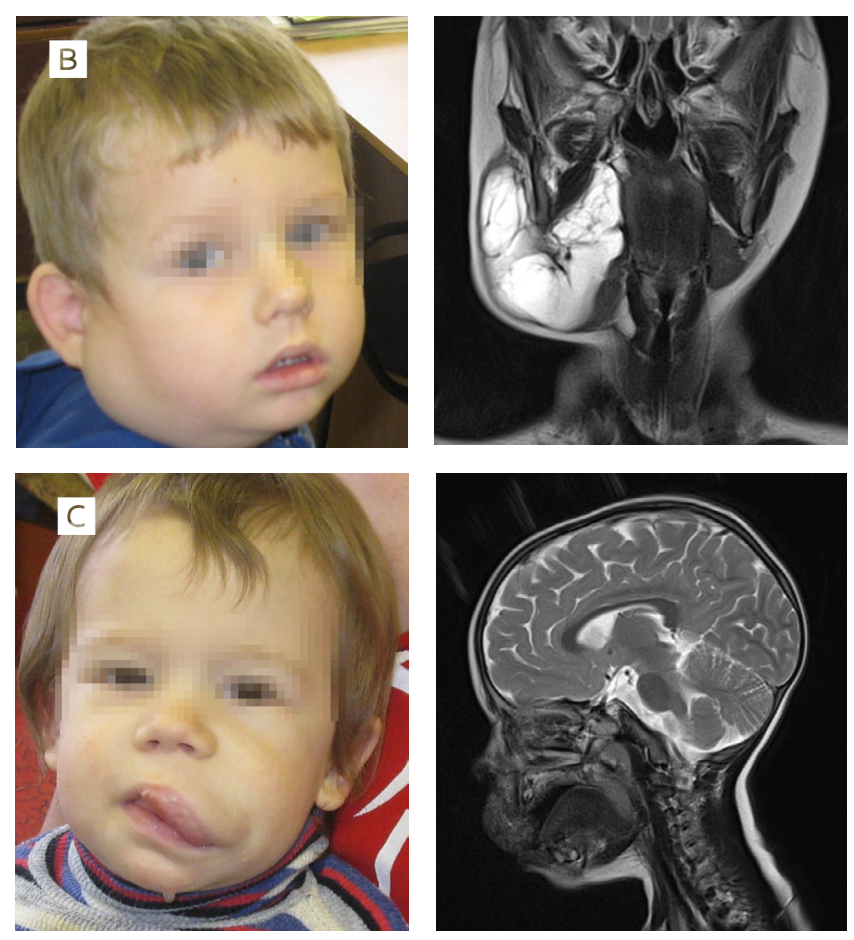

hyperintensity on T2 weighted and STIR (Short Tau Inversion Recovery) (18).

VM are accompanied by spontaneous thrombosis and thrombolysis $(19,20)$. Coagulation disorders are the result of chronic venous blood stagnation within the large irregular vascular spaces, leading to activation of coagulation cascade reactions following production of thrombin, the conversion of fibrinogen into fibrin, which induces the blood clot formation. (21). Following thrombosis, the process of thrombolysis starts, a reflection of which is to increase fibrin degradation products, including derived plasmin epitope D-dimer, which is called "a new diagnostic biomarker" to VM (22), because D-dimer level is increased in most patients with VM. In patients within our study group it increased in $82.4 \%$ of patients with VM. The newly formed microclots bind with plasma ionized calcium and form so-called "phleboliths", pathognomonic stone-like structures (23). According to published data, only half of LM have clinical signs at birth, $90 \%$ of LM are detected within the first two years of life, and the rest $10 \%$ are diagnosed at any age (24). In our clinical group, symptoms of LM appeared during the first two years of life in $85.7 \%$ of patients. Sometimes LM, especially if they are

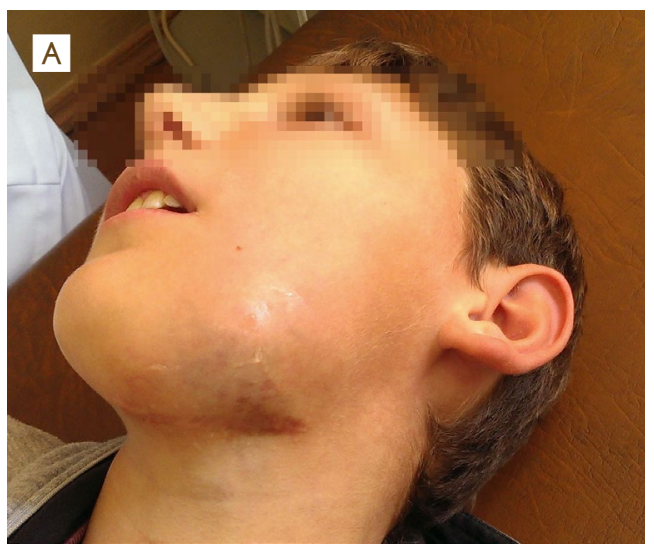

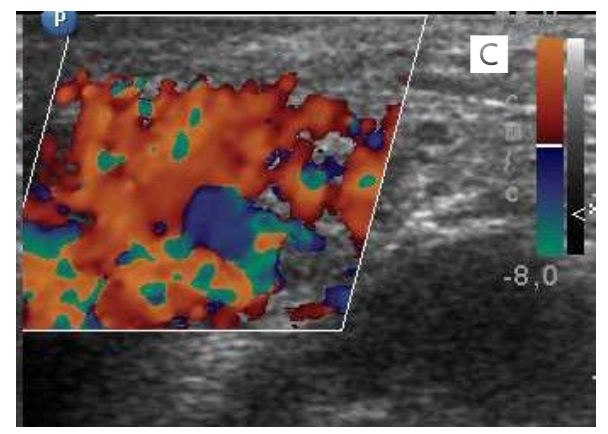

Fig. 8: Patient Z.: 11-years-old, AVM in mandible region. A - patient's photo (a hot, throbbing spot). B - US in gray scale mode - hyperechoid mass and hypoechoid areas. C- US in CDS mode - high flow vascularzed mass. 
large, can be detected during a prenatal screening ultrasound scan. The diagnosis was made prenatally in $10.7 \%$ of 112 children with LM. MRI is a routine diagnostic method in children with LM. At MRI it is possible to determine the structure of the mass, cyst size, thickness of membranes and conditional ratio of liquid, measure the borders of lesion, as well as determine the topographical relationships with the surrounding tissues. At MRI the boundaries between normal and affected soft tissue structures is clearly defined. Cysts filled with high protein fluid have high signal on T2, and are dark-color on T1. The most common is the classification of LM according the cyst's size: macrocystic, microcystic and mixed $(25,26)$. The size of cysts is relative, and in different data they range from 0.5 to 2 $\mathrm{cm}^{3}$. This division has practical importance. If the cyst can be successfully punctured and medication can be safety injected in its cavity, this LM is prognostically more favorable.

AVM are the congenital malformations of both arteri$\mathrm{al}$ and venous blood vessels, resulting in the formation of connections between vessels of different origin, diameter and resistance (27). These connections can be either direct or passing through the reticular structures, which is a net of dysplastic small vessels that have broken capillaries to maturation, and are called "nidus".

The most common AVM are those of central nervous system (CNS). Extracranial AVM are relatively rare, and their true prevalence in children is unknown. Liu A.S. at al. presents the largest group of patients with extracranial AVM (28), including 272 patients, both children and young adults. AVM percentage rate is $14.3 \%$ among patients with vascular malformations (29), but they rarely have clinical manifestation in the childhood age. In our pediatric group of patients with AVM, there $3.8 \%$ of patients with vascular malformations, who presented with clinical symptoms (usually bleeding). The primary symptoms of superficial AVM are visible in early childhood, but during the period of childhood in most patients significant changes and complications are not observed. In newborn children AVM visually present as pink or red spots, and at this stage any known methods of visualization cannot distinguish AVM and capillary spots (30). Children with the suspicion of AVM should be treated by performing basic non-invasive and minimally invasive techniques: US and Doppler mode and CT angiography with 3D reconstruction. Selective angiography should be performed in children immediately before treatment.

\section{CONCLUSIONS}

Adaptation and implementation of a uniform system of classification in everyday practice is the first step towards determining the correct diagnosis and treatment of VAs. In our gourp, like tumors and malformations, VAs were most commonly localized in the head and neck region. Clinical manifestations of VAs had clear age features. Vascular tumors among hospitalized patients made up $31.7 \%$, and the other $68.3 \%$ of patients had vascular malformations. Diagnosis of vascular malformations and rare vascular tumors should be made in a specialized center by using a diagnostic algorithm, to avoid erroneous diagnosis and to exclude more invasive diagnostic procedures.

\section{REFERENCES}

1. Hassanein AH, Mulliken JB, Fishman SJ, Greene AK. Evaluation of terminology for vascular anomalies in current literature. Plast Reconstr Surg 2011; 127(1): 347-51.

2. Puttgen K, Pearl M, Tekes A, Mitchell SE. Update on pediatric extracranial vascular anomalies of the head and neck. Childs Nerv Syst (2010); 26: 1417-33.

3. Dasgupta R, Fishman SJ. ISSVA classification. Seminars in Pediatric Surgery 2014; 23: 158-61.

4. Wassef M, Blei F, Adams D, et al. Vascular Anomalies Classification: Recommendations From the International Society for the Study of Vascular Anomalies. Pediatrics 2015; 136(1): e203-15.

5. Mulliken JB, Glowacki J. Hemangiomasand vascular malformations in infants and children: a classification based on endothelial characteristics. Plast Reconstr Surg 1982; 69: 412-22.

6. Konez O, Burrows PE. Magnetic resonance of vascular anomalies. Magn Reson Imaging Clin N Am 2002; 10(2): 363-88.

7. Greene AK, Liu AS, Mulliken JB, Chalache K, Fishman SJ. Vascular anomalies in 5621 patients: guidelines for referral. Journal of Pediatric Surgery 2011; 46:1784-89.

8. Dubois J, Garel L. Imaging and therapeutic approach of hemangiomas and vascular malformations in the pediatric age group. Pediatr Radiol 1999; 29: 879-93.

9. Eivazi B, Ardelean M, Bäumler W, et al. Update on hemangiomas and vascular malformations of the head and neck. European Archives of Oto-Rhino-Laryngology 2008; 266(2): 187-97.

10. Liang MG, Frieden IJ. Infantile and congenital hemangiomas. Seminars in Ped Surg 2014; 23: 162-7.

11. Donaldson JS. Pediatric vascular anomalies: the role of imaging and interventional radiology. Pediatr Ann 2008; 37: 414-24.

12. Ryan C, Price V, John P, et al. Kasabach-Merritt phenomenon: a single centre experience. Euerop J of Haematol 2010; 84(2): 97-104.

13. Croteau SE, Liang MG, Kozakewich HP, et al. Kaposiform hemangioendothelioma: atypical features and risks of Kasabach-Merritt phenomenon in 107 referrals. Pediatr 2013; 162(1): 142-7.

14. Dasgupta R, Patel M. Venous malformations. Seminars in Ped Surgery 2014; 23: 221-6.

15. Lee BB, Baumgartner I, Berlien P, et al. Diagnosis and treatment of venous malformations. Consensus Document of the International Union of Phlebology (IUP): Updated-2013. Int Angiol 2013; 34(2): 97-149.

16. McRae MY, Adams S, Pereira J, Parsi K, Wargon O. Venous malformations: clinical course and management of vascular birthmark clinic cases. Australas J Dermatol 2013 54: 22-30.

17. Upton J, Taghinia A Special considerations in vascular anomalies: operative management of upper extremity lesions. Clin Plast Surg $2011 ; 38(1): 143-51$.

18. Fayad LM, Fayad L, Hazirolan T, Bluemke D, Mitchell S. Vascular malformations in the extremities: emphasis on MR imaging features that guide treatment options. Skeletal Radiol 2006; 35: 127-137.

19. Dompmartin A, Acher A, Thibon P, et al. Association of Localized Intravascular Coagulopathy With Venous Malformations. Arch Dermatol 2008; 144(7): 873-7.

20. Mazoyer E, Enjolras O, Bisdorff A, Perdu J, Wassef M, Drouet L. Coagulation disorders in patients with venous malformation of limbs and trunk: a case series of 118 patients. Arch Dermatol 2008; 144: 861-7.

21. Redondo P, Aguado L, Marquina M, et al. Angiogenic and prothrombotic markers in extensive slow-flow vascular malformations: implications for antiangiogenic/antithrombotic strategies. British Journal of Dermatology 2010; 162: 350-6.

22. Weibel L. Vascular anomalies in children. Vasa 2011;40:439-47.

23. Hein KD, Mulliken JB, Kozakewich HP, Upton J, Burrows PE. Venous malformations of skeletal muscle. Plast Reconstr Surg 2002; 110(7): 1625-35.

24. Acevedo JL, Shah RK, Brietzke SE. Nonsurgical therapies for lymphangiomas: a systematic review. Otolaryngology Head and Neck Surgery $2008 ; 138(4): 418-24$.

25. Smith MC, Zimmerman MB, Burke DK,. Bauman NM, Sato Yu, Smith RJH. Efficacy and Safety of OK-432 Immunotherapy of Lymphatic Malformations. J Laryngoscope 2009; 119: 107-15.

26. Khunger N. Lymphatic Malformations: Current Status. J Cutan Aesthet Surg 2010; 3(3): 137-8. 
27. Uller W, Alomari AI, Richter GT. Arteriovenous malformations. Semin Pediatr Surg 2014; 23(4): 203-7.

28. Liu AS, Mulliken JB, Zurakowski D, Fishman SJ, Greene AK. Extracranial arteriovenous malformations: natural progression and recurrence after treatment. Plast Reconstr Surg 2010; 125(4): 85-94.
29. Leeb BB, Lardeo J, Neville R. Arterio-venous malformation: how much do we know? Phlebology 2009; 24: 193-200.

30. Paltiel HJ, Burrows PE, Kozakewich HP, Zurakowski D, Mulliken JB. Soft-tissue vascular anomalies: Utility of US for diagnosis. Radiology 2000; 214: 747-54. 